\title{
A new fluid structure coupling
}

\section{Application to parachute modelling}

\author{
Nicolas Aquelet* — Benjamin Tutt** \\ * Livermore Software Technology Corporation \\ 7374 Las Positas Road, Livermore, CA 94550, USA \\ aquelet@lstc.com \\ ** Irvin Aerospace Inc, Santa Ana, CA 92704 \\ BTutt@irvinaerospace.com
}

\begin{abstract}
The modelling of parachutes at Irvin Aerospace Inc. was based on the penalty Euler-Lagrange coupling method to compute the interaction between an Arbitrary Lagrange Euler formulation for the air flow and an updated Lagrangian finite element formulation for the canopy dynamics. This approach did not permit the effect of fabric porosity to be accounted for. In this paper, a new porosity Euler-Lagrange coupling models the fabric permeability by assessing the interaction forces based on the Ergun porous flow model. This paper provides validations for the technique when considering parachute applications and discusses the interest of this development to the parachute designer.

RÉSUMÉ. La modélisation de parachutes à Irvin Aerospace Inc. reposait sur la méthode de couplage Euler-Lagrange par pénalisation afin de calculer l'interaction entre une formulation ALE (Arbitrary Lagrange Euler) pour la dynamique de l'air et une formulation lagrangienne en éléments membranaires pour modéliser la canopée du parachute. Cette approche ne permettait pas de prendre en compte la perméabilité de la canopée. Dans ce papier, une nouvelle méthode de couplage Euler-Lagrange modélise les effets poreux en évaluant les forces d'interaction basées sur le modèle d'écoulement poreux d'Ergun. Ce papier présente des validations de la méthode en l'appliquant à des problèmes de parachute et discute l'intérêt de ce développement pour la conception de parachute.

KEYWORDS: parachute, permeability, penalty Euler-Lagrange coupling, porous coupling, ALE formulation, Ergun Equation.

MOTS-CLÉS: parachute, perméabilité, couplage Euler-Lagrange par pénalisation, couplage poreux, formulation ALE, équation d'Ergun.
\end{abstract}

DOI:10.3166/REMN.16.521-536৫ 2007 Lavoisier, Paris. Tous droits réservés

REMN - 16/2007. Fluid structure interaction, pages 521 to 536 


\section{Introduction}

Two forms of porosity are considered in parachute design: geometric porosity and fabric permeability. Geometric porosity is defined as the ratio of all open areas or physical gaps to the total canopy area. Fabric air-permeability is defined as the airflow through the canopy cloth in $\mathrm{CFM} / \mathrm{ft}^{2}$ (cubic feet per minute per square foot), at $1 / 2$ inch water pressure. When considering parachutes consisting of both geometric porosity and fabric permeability, a term referred to as equivalent porosity is often used. Parachute porosity, whether geometric or permeability based is an important matter in the design of a parachute canopy. It affects drag performance, stability and opening forces. Parachute drag performance, maximum oscillation angle, and opening forces all reduce with increasing porosity. In the majority of applications, the reduction in stability and opening forces is advantageous but the decrease in drag is not. Further, a parachute that is too porous will not open at all. The substitution of an impervious material with a highly permeable fabric can turn a parachute from a wandering sloth into a plummeting stabilizer. An accurate consideration of fabric permeability has long eluded the parachute designer. The permeability of the canopy is devised to limit the "wake recontact phenomenon" or better known as "canopy collapse". (Spahr et al., 1981) first discussed the phenomenon of wake recontact using wake momentum considerations. They noticed that if the deceleration of the canopy is too important, the axial velocities in the wake region may be much larger than the parachute ones, what allows the wake to catch up with the parachute and collapse it. Thus, blowing through a porous fabric to push the wake away is important to prevent the parachute from collapsing.

The numerical simulation of the porous parachute problem is a complex fluid structure interaction phenomenon. To appropriately simulate, and therefore understand and predict, this behavior requires an accurate method of assessing this complex relationship. Analysis of a parachute or a flow field without its associated partner is excluding the inherent interaction between the two. In this paper, the canopy permeability is taken into account by a new Euler-Lagrange coupling formulation (Benson, 2004; Aquelet et al., 2005). The parachute meshed by Lagrangian finite elements is immersed in an ALE grid, which modeled the air fluid flow. The fluid structure interaction force computed by an Euler-Lagrange coupling is based on the Ergun equation (Ergun, 1952). The Lagrangian finite element formulation uses a computational mesh that follows the material deformation. This approach is efficient and accurate for problems involving moderate deformations like the parachute or eventually flows that are essentially smooth. When this latter departs from this kind of smoothness, the ALE formulation must be used because the finite element mesh is independent from the material flow. This takes away all problems associated with distorted mesh that are commonly encountered with a Lagrangian approach. Thus the Euler Lagrange coupling using an ALE formulation for the fluid, is more suitable for solving parachute problems and more generally, fast transient porous fluid structure interaction problems. First, the ALE formulation is able to simulate fluid large deformations and second, the porous coupling can handle the interaction between the fluid and the parachute. A more detailed 
description of the method is presented through the following parts. First the ALE and Lagrangian governing equations for the fluid and parachute respectively are described together with boundary conditions. Then a description of the porous EulerLagrange coupling algorithm is presented. Further, the numerical method is validated by a porous parachute application with a comparison to experimental data. The numerical application in this paper focus on the terminal descent phase, for which experimental results are available in the database of Irvin Aerospace.

\section{Description of the fluid and structure problems}

The fluid is solved by using an ALE formulation (Benson, 1992) on a Cartesian grid that overlaps the porous structure, while this latter is discretised by Lagrangian shells based on the Belytschko-Lin-Tsay formulation (Belytschko et al., 1984).

\subsection{ALE description of Navier-Stokes equations}

For simplicity, the numerical simulations in this paper have been restricted to an Eulerian formulation for the fluid, although the formulation can be extended to an ALE formulation. The Eulerian formulation is a particular case of the ALE finite element formulation. Thus a general ALE point of view is first adopted to solve the Navier-Stokes equations before presenting the Eulerian formulation.

In the ALE description of motion, an arbitrary referential coordinate is introduced in addition to the Lagrangian and Eulerian coordinates (Hughes et al., 1981; Souli, 2000). The total time derivative of a variable $f$ with respect to a reference coordinate can be described as Equation [1]:

$$
\frac{d f(\vec{X}, t)}{d t}=\frac{\partial f(\vec{x}, t)}{\partial t}+(\vec{v}-\vec{w}) \cdot \overrightarrow{g r a d} f(\vec{x}, t)
$$

where $\vec{X}$ is the Lagrangian coordinate, $\vec{x}$ is the ALE coordinate, $\vec{v}$ is the particle velocity and $\vec{w}$ is the velocity of the reference coordinate, which will represent the grid velocity for the numerical simulation, and the system of reference will be later the ALE grid. Thus substituting the relationship between the total time derivative and the reference configuration time derivative derives the ALE equations.

Let $\Omega^{f} \in R^{3}$, represent the domain occupied by the fluid, and let $\partial \Omega^{f}$ denote its boundary. The equations of mass, momentum and energy conservation for a Newtonian fluid in ALE formulation in the reference domain, are given by:

$$
\frac{\partial \rho}{\partial t}+\rho \operatorname{div}(\vec{v})+(\vec{v}-\vec{w}) \operatorname{grad}(\rho)=0
$$




$$
\begin{aligned}
& \rho \frac{\partial \vec{v}}{\partial t}+\rho(\vec{v}-\vec{w}) \cdot \overline{\overline{\operatorname{grad}}}(\vec{v})=\overrightarrow{\operatorname{div}}(\overline{\bar{\sigma}})+\vec{f} \\
& \rho \frac{\partial e}{\partial t}+\rho(\vec{v}-\vec{w}) \cdot \overrightarrow{\operatorname{grad}}(e)=\overline{\bar{\sigma}}: \overline{\overline{\operatorname{grad}}}(\vec{v})+\vec{f} \cdot \vec{v}
\end{aligned}
$$

where $\rho$ is the density and $\overline{\bar{\sigma}}$ is the total Cauchy stress given by:

$$
\overline{\bar{\sigma}}=-p \cdot \overline{\overline{I d}}+\mu\left(\overline{\overline{\operatorname{grad}}}(\vec{v})+\overline{\overline{\operatorname{grad}}}(\vec{v})^{T}\right)
$$

where $p$ is the pressure and $\mu$ is the dynamic viscosity. Equations [2]-[4] are completed with appropriate boundary conditions. The part of the boundary at which the velocity is assumed to be specified is denoted by $\partial \Omega_{1}^{f}$. The inflow boundary condition is:

$$
\vec{v}=\vec{g}(t) \quad \text { on } \quad \partial \Omega_{1}^{f}
$$

The traction boundary condition associated with Equation [4] are the conditions on stress components. These conditions are assumed to be imposed on the remaining part of the boundary.

$$
\overline{\bar{\sigma}} \cdot \vec{n}=\vec{h}(t) \quad \text { on } \quad \partial \Omega_{2}^{f}
$$

One of the major difficulties in time integration of the ALE Navier-Stokes equations [2]-[4] is due to the nonlinear term related to the relative velocity $(\vec{v}-\vec{w})$. For some ALE formulations, the mesh velocity can be solved using a remeshing and smoothing process. In the Eulerian formulation, the mesh velocity $\vec{w}=\overrightarrow{0}$, this assumption eliminates the remeshing and smoothing process, but does not simplify the Navier-Stokes equations [2]-[4]. To solve equations [2]-[4], the split approach detailed in (Benson, 1992; Hughes, 1981) and implemented in most hydrocodes such as LS-DYNA ${ }^{\circledR}$ is adopted in this paper. Operator splitting is a convenient method for breaking complicated problems into series of less complicated problems. In this approach, first a Lagrangian phase is performed, using an explicit finite element method, in which the mesh moves with the fluid particle. In the CFD community, this phase is referred to as a linear Stokes problem. In this phase, the changes in velocity, pressure and internal energy due to external and internal forces are computed. The equilibrium equations for the Lagrangian phase are:

$$
\rho \frac{d \vec{v}}{d t}=\overrightarrow{\operatorname{div}}(\overline{\bar{\sigma}})+\vec{f}
$$




$$
\rho \frac{d e}{d t}=\overline{\bar{\sigma}}: \overline{\overline{\operatorname{grad}}}(\vec{v})+\vec{f} \cdot \vec{v}
$$

The mass conservation equation is used in its integrated form Equation [11] rather than as a partial differential equation (Belytschko et al., 2001). Although the continuity equation can be used to obtain the density in a Lagrangian formulation, it is simpler and more accurate to use the integrated form Equation [10] in order to compute the current density $\rho$ :

$$
\rho J=\rho_{0}
$$

where $\rho_{0}$ is the initial density and $J$ is the volumetric strain given by the Jacobian:

$$
J=\operatorname{det}\left(\frac{\partial x_{i}}{\partial X_{j}}\right)
$$

In the second phase, called advection or transport phase, the transportation of mass, momentum and energy across element boundaries are computed. This may be thought of as remapping the displaced mesh at the Lagrangian phase back to its initial position. The transport equations for the advection phase are:

$$
\begin{aligned}
& \frac{\partial \phi}{\partial t}+\vec{c} \cdot \overrightarrow{g r a d}(\phi)=0 \\
& \phi(\vec{x}, 0)=\phi_{0}(x)
\end{aligned}
$$

where $\vec{c}=\vec{v}-\vec{w}$ is the difference between the fluid velocity $\vec{v}$, and the velocity of the computational domain $\vec{w}$, which will represent the mesh velocity in the finite element formulation. In some papers (Hughes et al., 1981; Belytschko et al., 2001) $\vec{c}$ is referred as the convective velocity. The hyperbolic equation system [12] is solved by using a finite volume method. Either a first order upwind method or second order Van Leer advection algorithm (Van Leer, 1977) can be used to solve Equation [12]. The advection method is successively applied for the conservative variables: mass, momentum and energy with initial condition $\phi_{0}(\mathrm{x})$, which is the solution from the Lagrangian calculation of Equations [8]-[9] at the current time. In Equation 12, the time $t$ is a fictitious time: in this paper, time step is not updated when solving for the transport equation. There are different ways of splitting the Navier-Stokes problems. In some split methods, each of the Stokes problem and transport equation are solved successively for half time step. The following paragraph presents the description of the structure. 


\subsection{Lagrangian description of the porous structure}

In this paragraph the porous structure problem is described at the macroscopic scale and the Belytschko-Lin-Tsay shell formulation (Belytschko et al., 1984) is employed to model the parachute.

Let $\Omega^{s} \in R^{3}$, the domain occupied by the porous structure, and let $\partial \Omega^{s}$ denote its boundary. An updated Lagrangian finite element formulation is considered: the movement of the thin porous medium $\Omega^{s}$ described by $x_{i}(t),(i=1,2,3)$ can be expressed in terms of the reference coordinates $X_{i}(t),(i=1,2,3)$ and time t:

$$
x_{i}=x_{i}\left(X_{\alpha}, t\right)
$$

The momentum equation is given by Equation [16] in which $\overline{\bar{\sigma}}$ is the Cauchy stress, $\rho$ is the density, $f$ is the force density, $\frac{d \vec{v}}{d t}$ is acceleration and $\vec{n}$ is the unit normal oriented outward at the boundary $\partial \Omega^{s}$ :

$$
\begin{aligned}
& \rho \frac{d \vec{v}}{d t}=\overrightarrow{\operatorname{div}}(\overline{\bar{\sigma}})+\vec{f} \\
& \rho \frac{d e}{d t}=\overline{\bar{\sigma}}: \overline{\overline{\operatorname{grad}}}(\vec{v})+\vec{f} \cdot \vec{v}
\end{aligned}
$$

The solution of Equations [14]-[15] satisfies the displacement boundary condition Equation [16] on the boundary $\partial \Omega_{1}^{s}$ and the traction boundary condition Equation [17] on the boundary $\partial \Omega_{2}^{s}$.

$$
\begin{aligned}
& \vec{x}(\vec{X}, t)=\vec{D}(t) \text { on } \partial \Omega_{1}^{s} \\
& \overline{\bar{\sigma}} \cdot \vec{n}=\vec{\tau}(t) \text { on } \partial \Omega_{2}^{s}
\end{aligned}
$$

In this paper, the shell formulation used to model the parachute canopy is the Belytschko-Lin-Tsay formulation (Belytschko et al., 1984). The Belytschko-LinTsay shell 4-node element is based on a co-rotational coordinate system and a constitutive computation using a rate of deformation. The embedded element coordinate system that deforms with the element is defined in term of four corner nodes. As the element deforms, an angle may exist between the fiber direction and the unit normal of the element coordinate system. The magnitude of this angle is 
limited in order to keep a plane shell geometry. In this local system, the ReissnerMindlin theory gives the velocity of any point in the shell according to the velocity of mid-surface and the rotations of the element's fibers. Then the rates of deformation are computed at the center of the element. The new Cauchy stresses are computed by using the material model and by accounting for the incremental rotation, $\Delta \overline{\bar{R}}$, which is obtained by expressing the element base vectors at $\mathrm{t}(\mathrm{n}+1)$ in the local system at $t(n)$. Then, the element-centered resultant forces and moments are obtained by integrating the stresses through the thickness of the shell. The relations between these forces and moments and the local nodal forces and moments are obtained by performing the principle of virtual power with one point quadrature. Finally, the global nodal forces and moments are derived by using the transformation relations defined by the global components of the corotational unit vectors. The following section presents the porous Euler-Lagrange coupling method, which handles the fluid - porous structure problem.

\section{Fluid - porous structure interaction}

In an explicit time integration problem, the main part of the procedure in the time step is the calculation of the nodal forces. After computation of fluid and structure nodal forces, we compute the forces due to the coupling, these will only affect nodes that are on the fluid - porous structure interface. For each structure node, a depth penetration $\vec{d}$ is incrementally updated at each time step, using the relative velocity $\overrightarrow{v_{r e l}}$ at the slave and master node. For this coupling, the slave node is a structure mesh node, whereas the master node is not a fluid mesh node, it can be viewed as a fluid particle within a fluid element, with mass and velocity interpolated from the fluid element nodes using finite element shape functions. The location of the master node is also computed using the isoparametric coordinates of the fluid element. If $\vec{d}^{n}$ represents the penetration depth at time $t=t^{n}$, it is incrementally updated in Equation [18]:

$$
\vec{d}^{n+1}=\vec{d}^{n}+{\overrightarrow{v_{r e l}}}^{n+1 / 2} . \Delta t
$$

In Equation [18] $\vec{v}_{r e l}^{n+1 / 2}={\overrightarrow{v_{s}}}^{n+1 / 2}-{\overrightarrow{v_{f}}}^{n+1 / 2}$ in which the fluid velocity $\vec{v}_{f}$ is the velocity at the master node location and the structure velocity $\overrightarrow{v_{s}}$ is the velocity at the slave node location. The coupling acts only if penetration occurs, $\vec{n}_{s} \cdot \vec{d}^{n}<0$, where $\overrightarrow{n_{s}}$ is built up by averaging normals of structure elements connected to the 
structure node. The porous coupling forces are derived from the integration of the Ergun Equation (Ergun, 1952) on the shell volume:

$$
\frac{d p}{d \hat{z}}=a(\mu, \varepsilon) \overrightarrow{v_{\text {rel }} \cdot} \cdot \overrightarrow{n_{s}}+b(\rho, \varepsilon)\left(\overrightarrow{v_{r e l} \cdot} \cdot \overrightarrow{n_{s}}\right)^{2}
$$

in which $\hat{z}$ is the local position along the fiber direction of the shell element and $\varepsilon$ is the porosity: $\varepsilon=\frac{v_{\text {void }}}{v_{\text {total }}} . \mu$ and $\rho$ are the dynamic viscosity and density, respectively The coefficient $a(\mu, \varepsilon)$ is the reciprocal permeability of the porous shell or viscous coefficient. $b(\rho, \varepsilon)$ represents the inertia coefficient. For flows under very viscous conditions the second term in Equation [19], which represents the inertia effects drops out and the Blake-Kozeny equation for laminar flows in porous media is obtained. At high rates of flow it is the first term or viscous term, which drops out and the Blurke-Plummer equation for turbulent flows in porous media is obtained. For the parachute application the inertia effects should be preponderant. These coefficients can be derived from the Ergun theory:

$$
\begin{aligned}
& a=\frac{150 \mu(1-\varepsilon)^{2}}{D^{2} \varepsilon^{3}} \\
& b=\frac{1.75 \rho(1-\varepsilon)}{D \varepsilon^{3}}
\end{aligned}
$$

D is a characteristic length defined by: $D=\frac{6(1-\varepsilon) V}{S}$ with $\mathrm{V}$, the volume of the canopy and S, the "wetted" surface.

The Ergun equation describes the magnitude of porous flow velocity at a given differential pressure based upon two coefficients. These coefficients assume a constant porosity, not to be confused with a constant permeability. Porosity is a characteristic of the fabric, whereas permeability is a description of the flow velocity at a given condition. Many materials can be highly porous without being permeable. It should also be noted that the porosity of some fabrics can change significantly with applied load but in the applications of this paper, a constant and uniform porosity is assumed. Figure 1 displays historical permeability data (AFFDL-TR-78-151 report, 1978) of a common parachute cloth fabric, of particular interest is the widely used MIL-C-7020 Type III. 


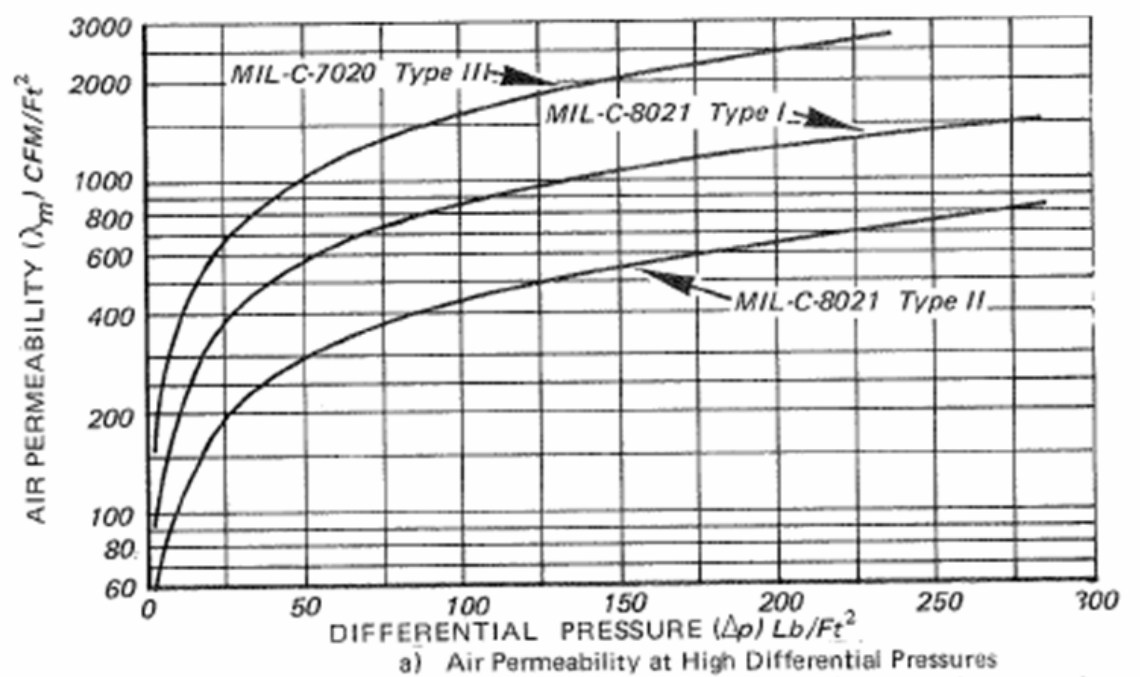

Figure 1. Parachute fabric permeability data (AFFDL-TR-78-151 report, 1978)

The data shown in Figure 1 was obtained at constant porosity, fluid viscosity and density. Under these assumptions the viscous and inertia parameters in Equation [19] are constant. To determine these coefficients, the Ergun theoretical permeability should be a parabolical fit of the experimental one. Thus the coefficients were computed by solving the following system:

$$
\left\{\begin{array}{l}
d p_{1} / e=a \cdot v_{1}+b \cdot v_{1}^{2} \\
d p_{2} / e=a \cdot v_{2}+b \cdot v_{2}^{2}
\end{array}\right.
$$

where $e$ is the shell thickness and the couple of points $\left(v_{1}, d p_{1}\right)$ and $\left(v_{2}, d p_{2}\right)$ was chosen on so that the Ergun equation fits the experimental plot as close as possible.

The force $F$ derived from Equation [19] is applied to both master and slave nodes in opposite directions to satisfy force equilibrium at the interface coupling, and thus the coupling is consistent with the fluid structure interface condition namely the action-reaction principle. At the structure coupling node, we applied a force:

$$
F_{s}=-F
$$


whereas for the fluid, the porous coupling force is distributed to the fluid element nodes based on the shape functions, at each node $\mathrm{i}(\mathrm{i}=1, . ., 8)$, the fluid force is scaled by the shape function $N_{i}$ :

$$
F_{f}^{i}=N_{i} \cdot F
$$

where $N_{i}$ is the shape function at node i. Since $\sum_{i=1}^{8} F_{f}^{i}=F$, the action-reaction principle is satisfied at the coupling interface. The following paragraph presents the validation of this approach to a parachute in terminal descent.

\section{Numerical application}

\subsection{Simulation methodology}

Before the development of the porous Euler-Lagrange coupling it was possible to analyse the parachute problem. A penalty Euler-Lagrange coupling algorithm permitted the interaction of the Eulerian formulation for the flow field, and the Lagrangian formulation for the parachute. Similarly to penalty contact algorithm (Belytschko et al., 1989), the coupling force in Equations [23]-[24] for the penalty Euler-Lagrange coupling is given by:

$$
F=k \cdot d
$$

where $k$ represents the spring stiffness, and $d$ the penetration computed by Equation [17]. The penalty Euler-Lagrange coupling was applied with the following methodology for parachute performance predictions at Irvin:

- model the parachute using a Lagrangian formulation,

- model the fluid domain using a Navier-Stokes based Eulerian formulation,

- perform the analyses using conditions similar to a wind tunnel, i.e. infinite mass flow; equating the results to the quasi-steady-descent phase of the parachute flight.

The last step reduced the computational cost associated with modelling vast spatial timelines associated with real parachute functions, specifically deployment and inflation. It also permitted the reduction in complexity of boundary conditions. Irvin developed this methodology several years ago and it has yielded excellent results for a number of parachutes with a low-permeability fabric. (Tutt, 2005) has previously published data that described the use of the penalty coupling to simulate the parachute behavior. This work discussed the benefit of visualizing the flying shape and anticipating the performance of a newly designed tactical mass assault troop parachute, prior to fabrication and testing. Particularly noteworthy is the replication of an undesirable flight characteristic exhibited by a replacement 
candidate for the venerable T-10 mass tactical assault parachute. The identification, and subsequent removal, of this flight mode through simulation design iterations demonstrated the powerful potential of such techniques. The modified version of that parachute system is now undergoing operational testing and will replace the $\mathrm{T}$ 10 later in this decade. Figure 2 illustrates a flight test with simulation flow field velocity vectors overlaid. The fabric shown in Figure 2 is classified as a lowpermeability fabric. When assessing the steady state characteristics of this parachute the approximation of an impermeable fabric was valid. Minimal differential pressure is developed across the canopy when a constant rate of descent is achieved.

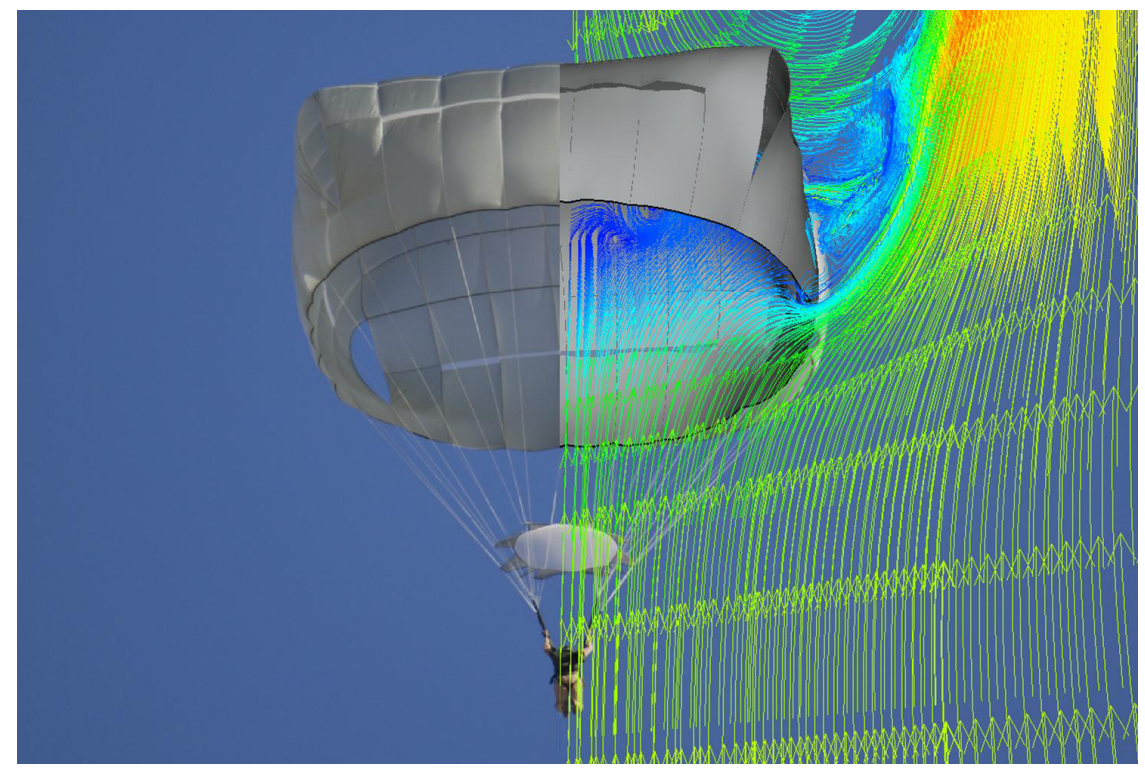

Figure 2. Flight test and simulation comparison

However the study in (Tutt, 2005) exposed also the inability to consider fabric permeability as an authentic limitation that would restrict the application of the methodology for a number of applications. To circumvent this drawback the porous Euler-lagrange coupling was developed and it now replaces the penalty coupling in the previous methodology. The following paragraph compares the two coupling method and shows the limits of the penalty coupling in solving parachute problems with a high-permeable canopy fabric.

\subsection{Parachute simulation}

A pertinent example of a parachute design that could not accurately be assessed using the penalty Euler-Lagrange coupling is the TP8 low altitude troop parachute. 
The TP8 is an aeroconical class of parachute. Aeroconical parachutes are commonly used for aircrew ejection systems. The TP8, shown in Figure 3, is fabricated from two base cloths of different permeability. The crown of the canopy is constructed from cloth exhibiting a permeability of $0.0508 \mathrm{~m} / \mathrm{s}\left(10 \mathrm{CFM} / \mathrm{ft}^{2}\right)$, and the major part of the skirt is rated at $0.4064 \mathrm{~m} / \mathrm{s}\left(80 \mathrm{CFM} / \mathrm{ft}^{2}\right)$, both at $1 / 2$ inch water pressure.

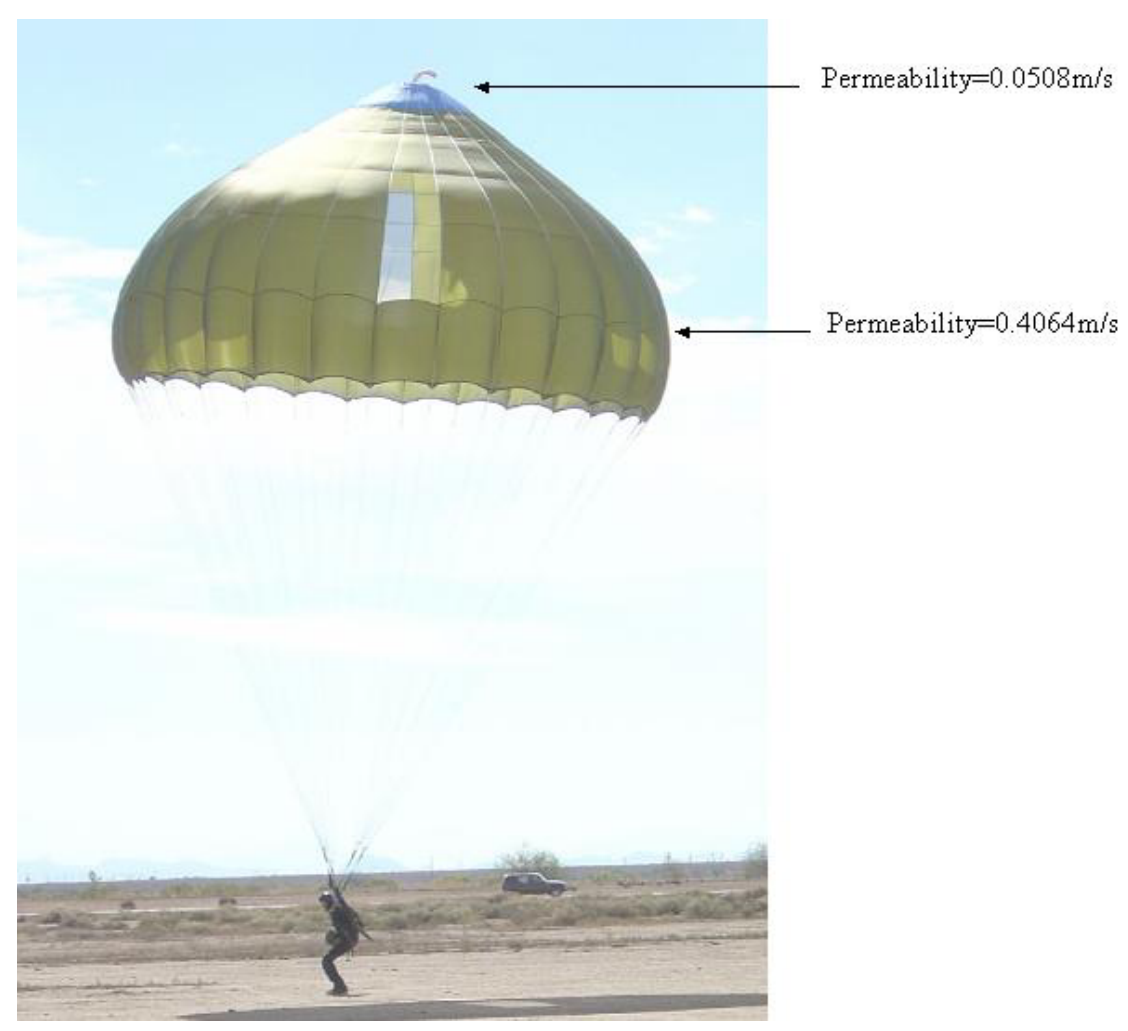

Figure 3. TP8 flight test

Drag coefficient, $C_{D}$, is widely used as a measure of parachute performance and is defined by:

$$
C_{D}=\frac{F_{D}}{1 / 2 \rho V^{2} S_{0}}
$$

where $F_{D}$ is the drag force, $S_{0}$ canopy surface area and $V$ is the velocity at the inlet of the channel. 
Test data indicates that the TP8 exhibits a drag coefficient of approximately 0.6. A constant flow rate is applied to the inlet of the wind tunnel modeled by the Eulerian grid. This flow rate equates to the steady rate of descent in an actual flight test. The parachute is modeled by a Lagrangian formulation. Two different simulations are compared with the experimental test:

- in the first modelling, the fluid structure interaction is handled by a penalty Euler-Lagrange coupling and,

- in the second one, the fluid structure interaction problem is solved with a porous Euler-Lagrange coupling.

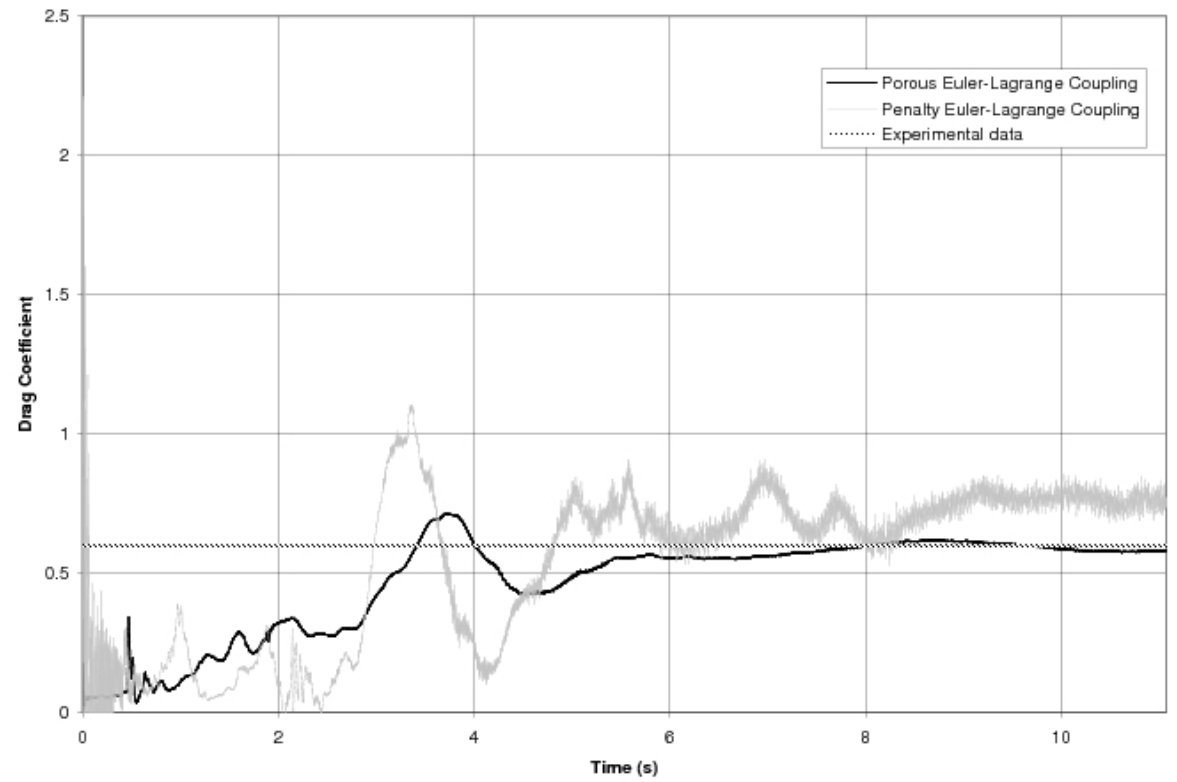

Figure 4. Drag force time history data

Figure 4 provides time history data of the drag force produced by the canopy when subjected to a flow velocity of $5.486 \mathrm{~m} / \mathrm{s}(18 \mathrm{ft} / \mathrm{s})$. On this figure, the coupling force time history reaches the steady state after $9 \mathrm{~s}$. The drag coefficient is computed after this time. The penalty coupling predicts a drag coefficient of 0.75 . Clearly, this prediction is in conflict with the experimental value of 0.6 , a value derived from a significant and reliable test series. The assumption of an impervious canopy cloth is the obvious factor in the difference between test and simulation results. Figure 5 presents a qualitative assessment of the permeability affect. It illustrates a crosssection of flow velocity for the TP8 simulation with and without accounting for fabric permeability. This figure presents an excellent illustration of the influence of fabric permeability in parachute design. The porous flow through the canopy cloth 
has completely changed the nature of the parachute wake. The large recirculation of air behind the parachute remains very close to the canopy on the left and has a significant effect on the parachute stability. By permitting air to flow through the canopy, the recirculating air has been pushed further downstream where it has considerably less influence on the stability of the parachute. Thus the "wake recontact phenomenon", which may cause the parachute collapse, is clearly prevented. This results in a discernible difference in drag coefficient. Figure 6 gives also a quantitative comparison of the same simulation with and without permeability. The data clearly depicts the reduction in drag associated with the incorporation of permeability. Also noticeable is the reduction in numerical noise, this is associated with a more benign parachute inflation, a characteristic of porous canopies. The steady-state drag force from Figure 4 can be used to calculate a modified drag coefficient; the simulation now predicts a $C_{d}$ of 0.59 , which is close to the experimental value: 0.6 .

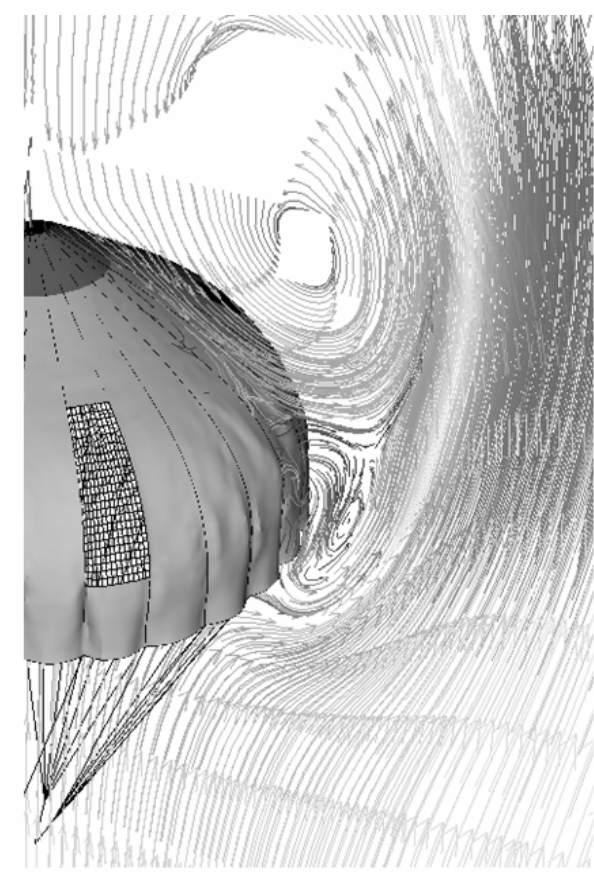

WITHOUT PERMEABILITY

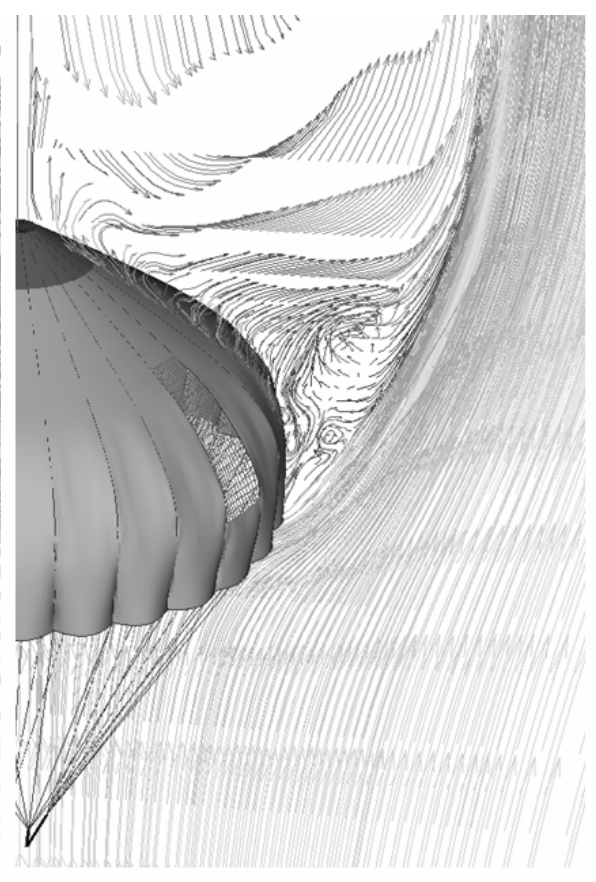

WITH PERMEABILITY

Figure 5. Visualization of the permeability effect 


\section{Conclusion}

The combination of bluff body aerodynamics and a highly deformable structure, fabricated from a porous media, creates a truly unique and multifaceted environment. To appropriately simulate, and therefore understand and predict, this behavior requires an accurate method of assessing this complex relationship. This paper has provided a description of the implementation and validation of the porous EulerLagrange coupling algorithm for a transient dynamic finite element method. It has also discussed the importance of such a development to the parachute engineer and the future of parachute design. Numerical results have been shown to provide excellent correlation with actual test data, providing an authentic capability to model parachutes fabricated from permeable fabrics. The prospective goals of this ongoing research are to incorporate the effect of fluid viscosity and particularly density changes during parachute flight. This will enable extremely high altitude and interplanetary aerodynamic decelerators to be evaluated over a range of conditions. Also of interest is the influence of fabric loading on porosity and the subsequent change in permeability.

\section{References}

Air Force Flight Dynamics Laboratory, Technical Report (AFFDL-TR-78-151), Recovery Systems Design Guide, June 1978.

Aquelet N., Souli M., Olovsson L., "Euler Lagrange Coupling with Damping Effects: Application to Slamming Problem", Comput. Methods Appl. Mech. Engrg., 2005, CMA5874, vol. 195, n 1-3, p. 110-132.

Belytschko T., Lin J., Tsay C.S., "Explicit algorithms for nonlinear dynamics of shells", Comp. Meth. Appl. Mech. Engrg., vol. 42, 1984, p. 225-251.

Belytschko T., Liu W.K., Moran B., Nonlinear Finite Elements for Continua and Structures, John Wiley \& Sons, LTD, 2001.

Belytschko T., Neal M.O., "Contact-impact by the pinball algorithm with penalty, projection, and Lagrangian methods", Proc. Symp. on Computational Techniques for Impact, Penetration, and Performation of Solids, ASME, New York, AMD-vol. 103, 1989, p. 97140.

Benson D., "Eulerian-Lagrangian Coupling in Finite Element calculations with applications to machining", Emerging Technology in Fluids, Structures, and Fluid-Structure Interactions 2004, vol. 2, July 25-29 2004, San Diego, California USA, PVP-vol. 485-2.

Benson D.J., "Computational methods in Lagrangian and Eulerian hydrocodes", Comp. Meth. Appl. Mech. Engrg., vol. 99, n 2, 1992, p. 235-394.

Ergun S., "Fluid flow through packed beds", Chem. Eng. Prog., vol. 48, n 2, 1952, p. 89-94.

Hughes T.J.R., Liu W.K., Zimmerman T.K., "Lagrangian Eulerian finite element formulation for viscous flows", Comp. Meth. Appl. Mech. Engrg., vol. 21, 1981, p. 329-349. 
Souli M., Ouahsine A., Lewin L., "ALE and Fluid-Structure Interaction problems", Comp. Meth. Appl. Mech. Engrg., vol. 190, 2000, p. 659-675.

Spahr H.R., Wolf D.E., "Theoretical analysis of wake-induced parachute collapse", $7^{\text {th }}$ AIAA Aerodyn. Decelerator and Balloon Technol. Conf., 1981, p. 81-1922.

Tutt B., "The use of LS-DYNA to Assess the Performance of Airborne Systems North America Candidate ATPS Main Parachutes", AIAA Aerodynamic Decelerator Systems Conference, 2005.

Van Leer B., "Towards the Ultimate Conservative Difference Scheme.IV. A New Approach to Numerical Convection", Journal Computational Physics, vol. 167, 1977, p. 276-299. 